\title{
Seletividade de Herbicidas. I - UTILIzaÇÃo do Método de Testemunhas Pareadas em Experimento com Cana-de-AçúCaR ${ }^{1}$
}

\author{
Herbicide Selectivity. I - Use of Additional Check Methods in Sugarcane Experiments
}

AZANIA, C.A.M. ${ }^{2}$, ROLIM, J.C. ${ }^{3}$, CASAGRANDE, A.A. ${ }^{4}$, LAVORENTI, N.A. ${ }^{5}$ e AZANIA, A.A.P.M. ${ }^{6}$

\begin{abstract}
RESUMO - Este trabalho objetivou comparar a precisão experimental dos delineamentos em blocos casualizados e blocos casualizados com testemunhas pareadas na avaliação da seletividade de herbicidas em soqueira de cana-de-açúcar de terceiro corte, cultivar RB835089. Os delineamentos utilizados foram o de blocos casualizados convencional e o de blocos casualizados com testemunhas pareadas, com quatro repetições. Os tratamentos foram diuron + hexazinone, azafenidin + hexazinone, metribuzin e isoxaflutole e testemunha capinada. Avaliaram-se na cana-de-açúcar a altura, o estande, o rendimento de colmos e o ATR (Açúcar Teórico Recuperável). O delineamento em blocos casualizados com testemunhas pareadas foi mais eficaz que aquele com testemunha tradicional. Constatou-se também que, para comparação das médias dos tratamentos, o teste $\mathrm{t}$ foi mais sensivel que o teste de Tukey, pois apresentou, com maior precisão experimental, mais tratamentos com diferenças em relação à testemunha.
\end{abstract}

Palavras-chave: cana-de-açúcar, herbicidas, seletividade, estatística, precisão experimental.

\begin{abstract}
This research aimed to evaluate the experimental precision of randomized complete block design and randomized complete block design using additional checks on herbicide selectivity using a third cut ratoon, $c v$ RB835089. The experimental designs consisted of a randomized complete block and a randomized complete block with additional checks in four replications. The treatments were diuron + hexazinone, azafenidin + hexazinone, metribuzin and isoxaflutole and hand-weeded checks. Sugarcane height, stand, weight and recoverable sugar were evaluated. The randomized complete block design with additional checks was more effective than the randomized complete block design. However, when comparing treatment averages, the Student's t test was more sensitive than the Tukey's test, since it presented more treatments with differences in relation to the check, with higher experimental precision.
\end{abstract}

Key words: sugarcane, herbicides, selectivity, statistics, and experimental precision.

\section{INTRODUÇÃO}

A maior parte dos herbicidas utilizados em cana-de-açúcar é seletiva, ou seja, controla as plantas daninhas sem comprometer o desenvolvimento e a produtividade da cultura. Essa seletividade é indicada pelas empresas de herbicidas a partir de ensaios, os quais procuram testar os novos herbicidas comparativamente com aqueles cujos efeitos são sabidamente conhecidos.
Esses ensaios de seletividade de herbicidas são instalados, no campo, geralmente no delineamento em blocos casualizados (DBC), com os resultados submetidos a análise de variância, aplicação do teste $\mathrm{F}$, seguido de um teste de comparação de médias (Tukey, teste t, Duncan etc.), quando necessário.

Ocorre que, para cana-de-açúcar, o uso de uma única testemunha dentro de cada bloco dificulta a observação de algumas variáveis,

1 Recebido para publicação em 11/4/2005 e na forma revisada em 25/11/2005.

Parte da tese de doutorado do primeiro autor apresentada à FCAVJ/UNESP.

2 Pqc. I Dr., Instituto Agronômico/Centro Cana, Rod. Anel Viário Contorno Sul, km 321, Caixa Postal 206, 14001-970 Ribeirão Preto-SP. ${ }^{3}$ Prof. Dr. do CCA/UFSCar; ${ }^{4}$ Prof. D.S. da FCAVJ/UNESP; ${ }^{5}$ Prof. Dr. do CCA/UFSCar; ${ }^{6}$ Pós-graduanda em Produção Vegetal (doutorado) da FCAVJ/UNESP. 
principalmente quando as plantas estão mais desenvolvidas. Essa dificuldade prejudica a precisão do experimento, podendo resultar em análises com falsas interpretações.

Na tentativa de aumentar a precisão experimental, diferentes delineamentos e procedimentos foram desenvolvidos para diferentes culturas. Nesse sentido, constatouse maior eficiência para o delineamento em blocos aumentados na cultura de batata (Bearzoti et al., 1997) e feijão (Souza et al., 2000); delineamento em látice, para soja (Lin et al., 1993); e precisão semelhante entre o DBC e o delineamento com testemunhas intercalares, para feijão (Faleiro et al., 2002).

A precisão experimental também pode ser aumentada utilizando-se de artifícios como o aumento no número de repetições e/ou a diminuição do tamanho das parcelas. Nesse caso, Resende \& Souza Júnior (1997), trabalhando com progênies de milho, verificaram que o aumento no número de repetições foi mais eficiente que o aumento do tamanho das parcelas na avaliação do progresso genético do milho. Em culturas perenes arbóreas, Rossetti (2002) verificou que a redução no tamanho das parcelas permitiu maior número de repetições, diminuindo o erro experimental e aumentando a precisão dos testes estatísticos aplicados aos dados observados.

Em plantas daninhas, uma das constatações de Velini (1995) foi de que o aumento da área amostrada por parcela foi mais eficiente do que o aumento do número de repetições do experimento, em termos de redução da variabilidade dos resultados. Entretanto, Palma (2000) comentou que o aumento do número de repetições não alterou os valores médios do quadrado médio do resíduo e do coeficiente de variação e o valor de $\mathrm{F}$ para os tratamentos. Também observou que o número de repetições tem um limite máximo, acima do qual os ganhos com precisão experimental são pequenos.

Em virtude da escassez de literatura sobre técnicas que aumentem a precisão experimental, especificamente em ensaios envolvendo seletividade de herbicidas, este trabalho teve como objetivo comparar a precisão experimental dos delineamentos em blocos casualizados convencional e blocos casualizados com testemunhas pareadas na avaliação da seletividade de herbicidas na cultura da cana-de-açúcar.

\section{MATERIAL E MÉTODOS}

O experimento foi instalado no município de Araras-SP, em condições de pós-emergência inicial da soqueira de cana-de-açúcar de terceiro corte, cultivar RB835089, em novembro de 2001 . O solo do experimento possui textura muito argilosa, apresentando $61 \%$ de argila, $24 \%$ de areia, $15 \%$ de silte e $21 \mathrm{~g} \mathrm{dm}^{-3}$ de matéria orgânica.

Os tratamentos foram constituídos pelos herbicidas diuron + hexazinone $(1.170$ + $\left.330 \mathrm{~g} \mathrm{ha}^{-1}\right)$, azafenidin + hexazinone $(192,5$ + 247,5 $\left.\mathrm{g} \mathrm{ha}^{-1}\right)$, metribuzin $\left(1,92 \mathrm{~L} \mathrm{ha}^{-1}\right)$, isoxaflutole $\left(127,5 \mathrm{~g} \mathrm{ha}^{-1}\right)$ e um tratamento testemunha; todos esses tratamentos foram mantidos capinados durante a realização do experimento. O delineamento experimental foi o de blocos casualizados com cinco tratamentos e quatro repetições, salientando-se que existia uma testemunha capinada para cada tratamento químico, dentro de cada bloco. As parcelas foram constituídas de cinco linhas de cana-de-açúcar com 10 m de comprimento, espaçadas de $1,30 \mathrm{~m}$, sendo consideradas úteis as três linhas centrais $\left(39 \mathrm{~m}^{2}\right)$. Na aplicação utilizou-se equipamento costal pressurizado $\left(\mathrm{CO}_{2}\right)$, munido de barra com três bicos Teejet DG (Drift Guardian) 110.02 VS, espaçados de $0,50 \mathrm{~cm}$, que, trabalhando a uma pressão de 35 libras pol$^{-2}$, proporcionou um volume de calda de $250 \mathrm{~L} \mathrm{ha}^{-1}$.

A seletividade dos herbicidas às plantas de cana-de-açúcar foi avaliada, na área útil de cada parcela, pela altura (do solo até dew lap) de 10 plantas escolhidas ao acaso aos 30, 45 e 60 DAT (dias após tratamento); pelo estande (contagem dos colmos) aos 180 DAT; pelo rendimento de colmos (t ha-1) e açúcar teórico recuperável\%caldo (ATR), sendo esta última característica determinada pela amostragem de 10 colmos na linha central de cada parcela e enviada a laboratório.

Os dados foram analisados de acordo com o delineamento em blocos casualizados, considerando-se quatro tratamentos químicos e uma testemunha (DBC com testemunha tradicional), bem como quatro tratamentos 
químicos e quatro testemunhas (DBC com testemunhas pareadas).

A análise dos dados para o DBC com testemunha tradicional foi obtida fazendo-se a média de todas as testemunhas de um mesmo bloco, obtendo-se apenas uma única testemunha por bloco. Neste caso, o teste F da análise de variância e os testes t e Tukey, para comparação de médias, foram obtidos de forma tradicional.

Entretanto, o teste $\mathrm{F}$ da análise de variância para o DBC com testemunhas pareadas foi obtido a partir de planilha própria elaborada no software Microsoft Excel, de acordo com a tabulação proposta na Tabela 1 e o esquema mostrado na Tabela 2 , sendo:

Soma de quadrados de blocos $=$

$$
\sum_{j=1}^{4} B^{2} / I-F C=\left(B_{1}^{2}+B_{2}^{2}+B_{3}^{2}+B_{4}^{2}\right) / I-F C
$$

em que $I=$ número de tratamentos.

Soma de quadrados de tratamentos $=$

$$
\left(\sum_{i=1}^{4} T_{i}\right)^{2} / j . r+\sum_{i=5}^{8} T_{i}^{2} / j-F C
$$

em que $T_{i}=$ total de tratamentos, $j=$ número de blocos, $r=$ número de testemunhas; e $I=$ número de tratamentos.

Soma de quadrados total $=$

$$
\sum_{i=1}^{8} \sum_{j=1}^{4} x_{i j}^{2}=x_{11}^{2}+x_{12}^{2}+\ldots+x_{84}^{2}
$$

Soma de quadrados do resíduo $=$

$$
S Q_{\text {resíduo }}=S Q_{\text {total }}-S Q_{\text {tratamentos }}-S Q_{\text {blocos }}
$$

Fator Correção $(F C)=$

$$
\left\{\left[\left(x_{11}+x_{12}+\ldots+x_{84}\right)^{2}\right] /\left(I_{. j}\right)\right\}
$$

em que $I=$ número de tratamentos; e $j=$ número de blocos.

Nos casos em que o valor de $\mathrm{F}$ da análise de variância apresentou-se significativo, utilizou-se o teste $\mathrm{t}$ de Student e o teste de Tukey para avaliação de diferenças entre médias. Os contrastes e as fórmulas utilizados para os testes $\mathrm{t}$ e Tukey encontram-se na Tabela 3. Todavia, para o DBC tradicional, ambos os testes foram calculados de acordo

Tabela 2 - Esquema de análise de variância utilizando-se o delineamento em blocos casualizados com testemunhas pareadas

\begin{tabular}{|l|c|l|c|c|}
\hline $\begin{array}{c}\text { Causas de } \\
\text { variação }\end{array}$ & GL & \multicolumn{1}{|c|}{$\mathrm{SQ}$} & $\mathrm{QM}$ & $\mathrm{F}$ \\
\hline Blocos & 3 & $\mathrm{SQ}_{\text {blocos }}$ & $\frac{S Q_{\text {blocos }}}{3}$ & $\frac{Q M_{\text {blocos }}}{Q M_{\text {residuo }}}$ \\
\hline Tratamentos & 4 & $\mathrm{SQ}_{\text {tratamentos }}$ & $\frac{S Q_{\text {tratamentos }}}{4}$ & $\frac{Q M_{\text {tratamentos. }}}{Q M_{\text {residuo }}}$ \\
\hline Resíduo & 24 & $\mathrm{SQ}_{\text {resíduo }}$ & $\frac{S Q_{\text {residuo }}}{24}$ & -- \\
\hline Total & 31 & $\mathrm{SQ}_{\text {total }}$ & --- & - \\
\hline
\end{tabular}

\begin{tabular}{|c|c|c|c|c|c|c|}
\hline \multicolumn{2}{|r|}{ Tratamento } & \multirow{2}{*}{$\frac{\text { Bloco } 1}{\mathrm{X}_{11}}$} & \multirow{2}{*}{$\frac{\text { Bloco } 2}{\mathrm{X}_{12}}$} & \multirow{2}{*}{$\frac{\text { Bloco } 3}{X_{13}}$} & \multirow{2}{*}{$\frac{\text { Bloco } 4}{X_{14}}$} & \multirow{2}{*}{$\frac{\text { Total }}{\sum T_{1}}$} \\
\hline $\mathrm{T} 1$ & Testemunha & & & & & \\
\hline $\mathrm{T} 2$ & Testemunha & $\mathrm{X}_{21}$ & $\mathrm{X}_{22}$ & $\mathrm{X}_{23}$ & $\mathrm{X}_{24}$ & $\sum T_{2}$ \\
\hline $\mathrm{T} 3$ & Testemunha & $\mathrm{X}_{31}$ & $\mathrm{X}_{32}$ & $\mathrm{X}_{33}$ & $\mathrm{X}_{34}$ & $\sum T_{3}$ \\
\hline $\mathrm{T} 4$ & Testemunha & $\mathrm{X}_{41}$ & $\mathrm{X}_{42}$ & $\mathrm{X}_{43}$ & $\mathrm{X}_{44}$ & $\sum T_{4}$ \\
\hline T5 & diuron + hexazinone & $\mathrm{X}_{51}$ & $\mathrm{X}_{52}$ & $\mathrm{X}_{53}$ & $\mathrm{X}_{54}$ & $\sum T_{5}$ \\
\hline T6 & azafenidin + hexazinone & $\mathrm{X}_{61}$ & $\mathrm{X}_{62}$ & $\mathrm{X}_{63}$ & $\mathrm{X}_{64}$ & $\sum T_{6}$ \\
\hline $\mathrm{T} 7$ & metribuzin & $\mathrm{X}_{71}$ & $\mathrm{X}_{72}$ & $\mathrm{X}_{73}$ & $\mathrm{X}_{74}$ & $\sum T_{7}$ \\
\hline $\mathrm{T} 8$ & isoxaflutole & $\mathrm{X}_{81}$ & $\mathrm{X}_{82}$ & $\mathrm{X}_{83}$ & $\mathrm{X}_{84}$ & $\sum T_{8}$ \\
\hline & Total & $\sum B_{1}$ & $\sum B_{2}$ & $\sum B_{3}$ & $\sum B_{4}$ & $\sum_{i=1}^{8} \sum_{j=1}^{4} x_{i j}$ \\
\hline
\end{tabular}

$\mathrm{GL}$ = graus de liberdade; $\mathrm{SQ}=$ soma de quadrados; $\mathrm{QM}=$ quadrado médio; $\mathrm{F}=$ teste $\mathrm{F}$.

Tabela 1 - Tabulação dos dados para análise de variância, de acordo com o delineamento em blocos casualizados com testemunhas pareadas 
Tabela 3 - Contrastes e fórmulas para o cálculo do teste t de Student e da diferença mínima significativa para o teste de Tukey, utilizando-se o delineamento em blocos casualizados com testemunhas pareadas

\begin{tabular}{|c|c|c|c|}
\hline \multicolumn{2}{|l|}{ Contraste } & \multirow{2}{*}{$\frac{\mathrm{t} \text { calculado }}{\hat{Y} / \sqrt{2 \cdot Q M_{\text {residuo }} / r . j . j}}$} & \multirow[t]{2}{*}{ Tukey calculado $(\Delta)$} \\
\hline 1- testemunha vs. Herbicidas & $\mathrm{Y}=4 \cdot \mathrm{m}_{\mathrm{t}}-\mathrm{m}_{5}-\mathrm{m}_{6}-\mathrm{m}_{7}-\mathrm{m}_{8}$ & & \\
\hline 2- testemunha $v s$. (diuron + hexazinone) & $Y=m_{t}-m_{5}$ & \multirow{4}{*}{$\hat{Y} / \sqrt{\left(\frac{1}{\mathrm{j}}+\frac{1}{\mathrm{j} \cdot \mathrm{r}}\right) \cdot Q M_{\text {residuo }}}$} & \multirow{4}{*}{$q \cdot \sqrt{\frac{1}{2}} \cdot\left(\frac{1}{\mathrm{j}}+\frac{1}{j \cdot r}\right) \cdot Q M_{\text {resíduo }}$} \\
\hline 3- testemunha $v s$. metribuzin & $\mathrm{Y}=\mathrm{m}_{\mathrm{t}}-\mathrm{m}_{7}$ & & \\
\hline 4- testemunha vs. (azafenidin + hexazinone) & $Y=m_{t}-m_{6}$ & & \\
\hline 5- testemunha $v s$. isoxaflutole & $\mathrm{Y}=\mathrm{m}_{\mathrm{t}}-\mathrm{m}_{8}$ & & \\
\hline 6- (diuron + hexazinone) vs. metribuzin & $\mathrm{Y}=\mathrm{m}_{5}-\mathrm{m}_{7}$ & \multirow{6}{*}{$\hat{Y /} \sqrt{\frac{2}{j} \cdot Q M_{\text {residuo }}}$} & \multirow{6}{*}{$q \cdot \frac{\sqrt{Q M_{\text {residuo }}}}{\sqrt{j}}$} \\
\hline 7-(diuron + hexazinone) vs. (azafenidin + hexazinone) & $Y=m_{5}-m_{6}$ & & \\
\hline 8-(diuron + hexazinone) vs. isoxaflutole & $Y=m_{5}-m_{8}$ & & \\
\hline 9- (azafenidin + hexazinone) $v s$. metribuzin & $Y=m_{6}-m_{7}$ & & \\
\hline 10- (azafenidin + hexazinone) vs. isoxaflutole & $Y=m_{6}-m_{8}$ & & \\
\hline 11- metribuzin $v s$. isoxaflutole & $\mathrm{Y}=\mathrm{m}_{7}-\mathrm{m}_{8}$ & & \\
\hline
\end{tabular}

$\mathrm{m}_{\mathrm{t}}=$ média da testemunha; $\mathrm{m}_{5}=$ média de diuron + hexazinone; $\mathrm{m}_{6}=$ média de azafenidin + hexazinone; $\mathrm{m}_{7}=$ média de metribuzin; $\mathrm{m}_{8}=$ média de isoxaflutole; $\Delta=$ diferença mínima significativa; $r=$ número de repetições da testemunha; $j=$ número de blocos; QMres $=$ quadrado médio do resíduo; valores de t tabelado: 2,80 (0,01) e 2,06 (0,05); $q=$ amplitude total estudentizada: $4,17(0,05)$.

com a literatura, podendo ser executado em qualquer programa estatístico; para o teste $t$, os contrastes estabelecidos foram os mesmos apresentados na Tabela 3.

A precisão experimental entre o DBC com testemunha tradicional e o DBC com testemunhas pareadas foi avaliada utilizando-se o erro-padrão da diferença entre a média de testemunha e a média de herbicida, sendo para o DBC tradicional:

$$
s(\hat{y})_{D B C \text { testemunha tradicional }}=\sqrt{2 \cdot Q M_{\text {resíduo }} / j}
$$

e, para testemunhas pareadas:

$$
s(\hat{y})_{D B C \text { testemunhas pareadas }}=\sqrt{\left(\frac{1}{j}+\frac{1}{r \cdot j}\right) \cdot Q M_{\text {residuo }}}
$$

em que $j=$ número de blocos; e $r=$ número de testemunhas.

\section{RESULTADOS E DISCUSSÃO}

\section{Teste F para análise de variância}

Na Tabela 4 observa-se que o DBC com testemunhas pareadas acusou, pelo teste $\mathrm{F}$ da análise de variância, um número maior de variáveis com diferenças entre os tratamentos quando comparado ao DBC com testemunha tradicional.

Planta Daninha, Viçosa-MG, v. 23, n. 4, p. 661-667, 2005
O maior número de variáveis com diferenças no DBC com testemunhas pareadas deve-se ao maior número de testemunhas, que proporcionou a elevação do número de grau de liberdade do resíduo (Tabela 2). Segundo Barbosa (2000), o grau de liberdade do resíduo é usado como denominador no cálculo do quadrado médio do resíduo, que, por sua vez, na análise de variância, é usado também como denominador para calcular o valor de $\mathrm{F}$.

No entanto, quanto maior o número de grau de liberdade do resíduo, espera-se que menor seja o quadrado médio do resíduo e, conseqüentemente, maior será o valor calculado no teste F. Quanto maior o valor de $\mathrm{F}$ calculado, maior será a possibilidade de rejeição da hipótese de nulidade, dentro dos níveis de probabilidade de 1 e $5 \%$.

Observando os valores de F pelo DBC com testemunha tradicional (Tabela 4), constatase que, com exceção da altura (45 DAT), as demais variáveis não foram afetadas pelos tratamentos, sendo não-significativas (NS); logo, aceitou-se a hipótese de que as médias dos tratamentos eram iguais estatisticamente. Assim, as plantas não foram prejudicadas quanto a perfilhamento (estande), rendimento de colmos e quantidade de açúcar (ATR). Contudo, quando se observaram as mesmas variáveis sendo submetidas à análise de variância em DBC com testemunhas pareadas, além da altura (45 DAT), também se encontrou 
Tabela 4 - Valores calculados do teste F, coeficientes de variação (CV) e erro-padrão da diferença entre duas médias de tratamentos para as variáveis altura, estande, produção e açúcar teórico recuperável (ATR), obtidas dos delineamentos em blocos casualizados com testemunha tradicional e pareada, em experimento de aplicação de herbicida na pós-emergência inicial da cana-de-açúcar, cultivar RB835089, durante a estação chuvosa (safra 2001/02). Araras-SP, 2003

\begin{tabular}{|c|c|c|c|c|c|c|c|c|}
\hline \multirow[t]{2}{*}{ Aplicação } & \multirow[t]{2}{*}{ Variável } & \multirow{2}{*}{$\begin{array}{l}\text { Época } \\
\text { (DAT) }\end{array}$} & \multicolumn{3}{|c|}{$\begin{array}{c}\text { DBC } \\
\text { Testemunha tradicional }\end{array}$} & \multicolumn{3}{|c|}{$\begin{array}{c}\text { DBC } \\
\text { Testemunha pareada }\end{array}$} \\
\hline & & & $\mathrm{F}$ & $\mathrm{CV}(\%)$ & $s(\hat{y})$ & $\mathrm{F}$ & $\mathrm{CV}(\%)$ & $s(\hat{y})$ \\
\hline \multirow{6}{*}{ Pós-inicial } & \multirow{3}{*}{ Altura $(\mathrm{cm})$} & 30 & $0,62 \mathrm{NS}$ & 7,80 & 2,00 & $1,20 \mathrm{NS}$ & 7,06 & 1,45 \\
\hline & & 45 & $3,88 *$ & 5,43 & 2,00 & $4,41 * *$ & 6,20 & 1,84 \\
\hline & & 60 & $0,38 \mathrm{NS}$ & 11,38 & 5,24 & $0,82 \mathrm{NS}$ & 11,34 & 4,23 \\
\hline & Estande $\left(\right.$ colmos $\left.\mathrm{m}^{-1}\right)$ & 180 & $3,04 \mathrm{NS}$ & 3,64 & 0,32 & $3,65 *$ & 3,94 & 0,27 \\
\hline & Rendimento $\left(\mathrm{kg} \mathrm{ha}^{-1}\right)$ & 273 & $2,07 \mathrm{NS}$ & 9,17 & 8,24 & $4,11 *$ & 7,20 & 5,20 \\
\hline & $\operatorname{ATR}^{1 /}\left(\mathrm{t}\right.$ açúcar ha $\left.{ }^{-1}\right)$ & 273 & $0,85 \mathrm{NS}$ & 5,10 & 4,95 & $1,06 \mathrm{NS}$ & 4,67 & 3,59 \\
\hline
\end{tabular}

DAT $=$ dias após tratamento; $\mathrm{DBC}=$ delineamento em blocos casualizados; $\mathrm{CV}=$ coeficiente de variação; $*$ significativo a $5 \%$ de probabilidade; ** significativo a $1 \%$ de probabilidade; $N S=$ não-significativo; $\mathrm{s}(\hat{\mathrm{y}})$ = erro-padrão da diferença entre a média de testemunha e a média de herbicida). ${ }^{1 /}$ Análise realizada pelo Laboratório de Sacarose da AFOCAPI.

significância estatística nos valores de $\mathrm{F}$ para o estande (180 DAT) e rendimento de colmos (273 DAT).

Essas maiores significâncias podem ser atribuídas aos menores valores do erropadrão da diferença, também observados pela Tabela 4 no DBC com testemunhas pareadas. Quando são comparados os DBCs entre testemunha tradicional e pareada, constata-se $8,00,15,63$ e $36,89 \%$ a menos de erro-padrão da diferença para altura (45 DAT), estande (180 DAT) e rendimento (273 DAT), respectivamente. Esses resultados sugerem que, à medida que o erro-padrão da diferença diminui, o erro experimental também diminui, aumentando a precisão experimental no DBC com testemunha pareada.

Observa-se também pela Tabela 4 que, para todos os experimentos, os valores do coeficiente de variação não foram bruscamente alterados pelo uso do DBC com testemunhas pareadas. Segundo Barbosa (2000), o valor para o coeficiente de variação deve ser inferior a $20 \%$, para experimentos de campo. Portanto, pode-se considerar, em função dos valores do coeficiente de variação, que os dados dos experimentos não apresentaram demasiada variação.

\section{Testes para comparação de médias}

As variáveis cujo teste $\mathrm{F}$ foi significativo tiveram as médias (Tabela 5) de seus tratamentos comparadas pelo teste t de Student e pelo teste de Tukey. Todavia, verificou-se que, para todas as variáveis submetidas a ambos os testes, o teste $t$ indicou um número maior de tratamentos com diferenças estatísticas que o teste de Tukey, tanto para o DBC com testemunha tradicional como para o DBC com testemunhas pareadas.

Nesse sentido, pode-se observar pela Tabela 6 que, para o DBC tradicional, o teste de Tukey apenas acusou para a altura (45 DAT) diferença no tratamento com isoxaflutole (contraste 4), enquanto o teste $\mathrm{t}$ acusou diferença nos contrastes $3,4,6,7$ e 10. Para o DBC com testemunhas pareadas pode-se observar que para altura (45 DAT) o teste $t$ também foi mais eficaz que o de Tukey, por ter acusado maior número de contrastes com diferenças significativas. Do mesmo modo, para o estande (180 DAT), o teste de

Tabela 5 - Médias de tratamentos para as variáveis altura, estande e produção obtida no experimento, a partir da aplicação de herbicida na pós-emergência inicial da canade-açúcar, cultivar RB835089, durante a estação chuvosa (safra 2001/02). Araras-SP, 2003

\begin{tabular}{|l|c|c|c|}
\hline Tratamento & $\begin{array}{c}\text { Altura }(\mathrm{cm}) \\
\text { 45 DAT }\end{array}$ & $\begin{array}{c}\text { Estande } \\
\left(\begin{array}{c}\left.\text { colmos } \mathrm{m}^{-1}\right) \\
180 \mathrm{DAT}\end{array}\right.\end{array}$ & $\begin{array}{c}\text { Rendimento } \\
\text { de colmos } \\
\left(\mathrm{t} \mathrm{ha}^{-1}\right) \\
273 \text { DAT }\end{array}$ \\
\hline Testemunha* & 55,03 & 12,19 & 133,39 \\
\hline Velpar** & 53,93 & 12,26 & 126,31 \\
\hline Sencor*** & 52,93 & 13,10 & 131,09 \\
\hline Evvolus*** & 49,43 & 12,83 & 131,73 \\
\hline Provence** & 48,75 & 12,36 & 112,79 \\
\hline
\end{tabular}

* média de 16 repetições; ** média de quatro repetições.

Planta Daninha, Viçosa-MG, v. 23, n. 4, p. 661-667, 2005 
Tabela 6 - Valores calculados para o teste de Tukey e teste t e respectivas significâncias sobre altura, estande e produção da cana-de-açúcar influenciada pela aplicação de herbicidas, em pós-emergência inicial da soqueira, cultivar RB835089, durante a estação chuvosa (safra 2001/02). Araras-SP, 2003

\begin{tabular}{|c|c|c|c|c|c|c|c|c|c|}
\hline \multirow{3}{*}{ № } & \multirow{3}{*}{ Contraste } & \multirow{2}{*}{\multicolumn{2}{|c|}{$\begin{array}{c}\text { DBC tradicional } \\
\text { Altura (45 DAT) }\end{array}$}} & \multicolumn{6}{|c|}{ DBC com testemunha pareada } \\
\hline & & & & \multicolumn{2}{|c|}{ Altura (45 DAT) } & \multicolumn{2}{|c|}{ Estande (180 DAT) } & \multicolumn{2}{|c|}{ Rendimento (273 DAT) } \\
\hline & & Tukey & $\mathrm{t}$ & Tukey & $\mathrm{t}$ & Tukey & $\mathrm{t}$ & Tukey & $\mathrm{t}$ \\
\hline 1 & Test. $v s$. (diuron+hexazinone) & & & & & $-0,07 \mathrm{NS}$ & $-0,24 \mathrm{NS}$ & $7,08 \mathrm{NS}$ & $1,36 \mathrm{NS}$ \\
\hline 2 & Test. $v s$. metribuzin & $2,10 \mathrm{NS}$ & $1,05 \mathrm{NS}$ & $2,10 \mathrm{NS}$ & $1,14 \mathrm{NS}$ & $-0,91 *$ & $-3,33 * *$ & $2,30 \mathrm{NS}$ & $0,44 \mathrm{NS}$ \\
\hline 3 & Test. vs. (azafenidin+hexazinone) & $5,60 \mathrm{NS}$ & $2,80 * *$ & $5,60 *$ & $3,04 * *$ & $-0,64$ NS & $-2,35 *$ & $1,66 \mathrm{NS}$ & $0,32 \mathrm{NS}$ \\
\hline 4 & Test. $v s$. isoxaflutole & $6,28 *$ & $3,14 * *$ & $6,28 *$ & $3,41 * *$ & $-0,17 \mathrm{NS}$ & $-0,61 \mathrm{NS}$ & $20,60 *$ & $3,96 * *$ \\
\hline 5 & (diuron+hexazinone) $v s$. metribuzin & $1,00 \mathrm{NS}$ & $0,50 \mathrm{NS}$ & $1,00 \mathrm{NS}$ & $0,43 \mathrm{NS}$ & $-0,84 \mathrm{NS}$ & $-2,44 *$ & $-4,78 \mathrm{NS}$ & $-0,73 \mathrm{NS}$ \\
\hline 6 & (diuron+hexazinone) vs. (azafenidin+hexazinone) & $4,50 \mathrm{NS}$ & $2,25 *$ & $4,50 \mathrm{NS}$ & $1,93 \mathrm{NS}$ & $-0,57 \mathrm{NS}$ & $-1,66 \mathrm{NS}$ & $-5,42 \mathrm{NS}$ & $-0,82 \mathrm{NS}$ \\
\hline 7 & (diuron+hexazinone) $v s$. isoxaflutole & $5,18 \mathrm{NS}$ & $2,59 *$ & $5,18 \mathrm{NS}$ & $2,22 *$ & $-0,10 \mathrm{NS}$ & $-0,29 \mathrm{NS}$ & $13,53 \mathrm{NS}$ & $2,05 \mathrm{NS}$ \\
\hline 8 & (azafenidin+hexazinone) vs. metribuzin & $-3,50 \mathrm{NS}$ & $-1,75 \mathrm{NS}$ & $-3,50 \mathrm{NS}$ & $-1,50 \mathrm{NS}$ & $-0,27 \mathrm{NS}$ & $-0,77$ NS & $0,64 \mathrm{NS}$ & $0,10 \mathrm{NS}$ \\
\hline 9 & (azafenidin+hexazinone) vs. isoxaflutole & $0,67 \mathrm{NS}$ & $0,34 \mathrm{NS}$ & $0,67 \mathrm{NS}$ & $0,29 \mathrm{NS}$ & $0,48 \mathrm{NS}$ & $1,37 \mathrm{NS}$ & $18,94 \mathrm{NS}$ & $2,88 * *$ \\
\hline 10 & metribuzin $v s$. Isoxaflutole & $4,18 \mathrm{NS}$ & $2,09 *$ & $4,18 \mathrm{NS}$ & $1,79 \mathrm{NS}$ & $0,74 \mathrm{NS}$ & $2,15 *$ & $18,30 \mathrm{NS}$ & $2,78 *$ \\
\hline & & 5,89 & $\underline{3}$ & $\begin{array}{l}5,43^{1 / 1} \\
6,87^{2 !}\end{array}$ & $\underline{3} /$ & $\begin{array}{l}0,81^{1 / 1} \\
1,02^{2 /}\end{array}$ & 3/ & $\begin{array}{l}15,35^{1 /} \\
19,41^{2 /}\end{array}$ & $\underline{3} /$ \\
\hline
\end{tabular}

$\mathrm{DBC}=$ delineamento em blocos casualizados; DAT $=$ dias após tratamento; $*$ significativo a $5 \%$ de probabilidade; $* *$ significativo a $1 \%$ de probabilidade; $\Delta=$ diferença mínima significativa; ${ }^{1 /} \Delta$ para média de tratamento testemunha $v$ s. herbicidas; ${ }^{\stackrel{2}{ }} \Delta$ para média entre tratamentos herbicidas; $\mathrm{e}^{3 / /}$ valores tabelados de (t): 2,06 (5\%) e 2,80 (1\%).

Tukey acusou diferenças entre testemunhas e metribuzin (contraste 2), ao passo que o teste $\mathrm{t}$, além dessa diferença, também foi mais sensivel à comparação entre testemunhas e azafenidin + hexazinone (contraste 3 ) e entre os herbicidas diuron + hexazinone e isoxaflutole (contrastes 6 e 10). Um outro exemplo de eficácia do teste $\mathrm{t}$ pode ser observado sobre o rendimento de colmos aos 273 DAT, entre o tratamento testemunha e o isoxaflutole (contraste 4), quando o teste $\mathrm{t}$ acusou maior precisão experimental $(0,01)$ que o Tukey $(0,05)$.

O teste de Tukey mostrou ser mais eficaz para o DBC com testemunhas pareadas. Essa melhor eficácia pode estar relacionada ao fato de o aumento no número de testemunhas ter possibilitado o cálculo de duas diferenças mínimas significativas (D): uma para os contrastes entre a média da testemunha e as médias dos herbicidas e outra para os contrastes entre tratamentos herbicidas.

Entretanto, o teste de Tukey, mesmo com melhor eficácia para o DBC com testemunhas pareadas, não foi mais eficaz que o teste $t$, que sempre acusou diferenças para um número maior de tratamentos ou para um mesmo número de tratamentos, porém com maior precisão experimental.

Assim, perante a análise dos dados, pode-se concluir que o DBC com testemunhas pareadas foi mais eficaz que o DBC com testemunha tradicional porque apresentou menor erro experimental e o teste $\mathrm{F}$ da análise de variância acusou mais variáveis com diferenças entre tratamentos. Verificou-se também que, para comparação das médias dos tratamentos, o teste $t$ foi mais sensivel que o teste de Tukey, pois apresentou mais tratamentos com diferenças em relação à testemunha.

\section{LITERATURA CITADA}

BARBOSA, J. C. Estatística experimental. Jaboticabal: 2000. 288 p.

BEARZOTI, E.; PINTO, C. A. B. P.; OLIVEIRA, M. S. Comparação entre métodos estatísticos na avaliação de clones de um programa de melhoramento de batata. Pesq. Agropec. Bras., v. 32, n. 9, p. 877-884, 1997.

FALEIRO, F. G. et al. Comparação de blocos casualizados e testemunha intercalares na estimativa de parâmetros genéticos em feijoeiro. Pesq. Agropec. Bras., v. 37, n. 12, p. 1675-1680, 2002.

LIN, C. S. et al. Performance of randomized block designs in field experiments. Agron. J., v. 85, n. 1, p. 168-171, 1993.

PALMA, V. Períodos de controle de plantas daninhas na cultura do milho (Zea mays L.). Efeito do número de repetições sobre a precisão experimental. 2000. 82 f. Tese (Doutorado em Agricultura) - Universidade Estadual Paulista, Botucatu, 2000. 
RESENDE, M. D. V.; SOUZA JUNIOR, C. L. Número de repetições e tamanho da parcela para seleção de progênies de milho em solos sob cerrado e fértil. Pesq. Agropec. Bras., v. 32, n. 9 , p. 148-156, 1997.

ROSSETTI, A. G. Influência da área da parcela e do número de repetições na precisão de experimentos com arbóreas. Pesq. Agropec. Bras., v. 37, n. 4, p. 433-438, 2002.
SOUZA, E. A.; GERALDI, I. O.; RAMALHO, M. A. P. Alternativas experimentais na avaliação de famílias em programas de melhoramento genético do feijoeiro. Pesq. Agropec. Bras., v. 35, n. 9, p. 1765-1771, 2000.

VELINI, E. D. Estudo e desenvolvimento de métodos experimentais e amostrais adaptados a matologia. 1995. 250 f. Tese (Doutorado em Produção Vegetal) - Universidade Estadual Paulista, Jaboticabal, 1995. 